\title{
Low-Dimensional Structures of Diluted Magnetic (Semimagnetic) Semiconductors - a Subjective Review
}

\author{
J. KossuT \\ Institute of Physics of the Polish Academy of Sciences and the College of Science \\ al. Lotników 32/46, 02-668 Warsaw, Poland
}

(Received November 5, 2001)

\begin{abstract}
A review on selected areas of research on low-dimensional structures consisting of diluted magnetic (semimagnetic) semiconductors is attempted. In the introductory part, after short historical preliminaries, we describe some early ideas and speculations concerning properties of such structures and we confront them with real results obtained in the course of their experimental investigations. Several issues are addressed to but, in view of the richness of the field, the review is by no means not pretending to be an exhaustive one. We begin with early studies of metal-insulator-semiconductor structures and progress to structures grown by molecular beam epitaxy. We discuss, in particular, the idea of a spin superlattice, type-I-to-type-II transition, wave functions mapping, and detailed determination of the profiles of realistic quantum wells. We put special emphasis on digital growth and, specifically, parabolic and half-parabolic quantum wells, use of tunable $g$-factor in studies of trions, self-assembled quantum dots, spin injection and, finally, on hybrid structures of ferromagnets and diluted magnetic semiconductor structures. The focus of the present paper is on work done in the Institute of Physics of the Polish Academy of Sciences, mostly in the Laboratory of Growth and Physics of Low-Dimensional Crystals.
\end{abstract}

PACS numbers: 78.20.-e, 78.67.-n, 75.70.-i

\section{Introduction and scope}

Diluted magnetic semiconductors (DMSs), known also as semimagnetic semiconductors (the term coined by R.R. Gałąza in the Institute of Physics of the Polish Academy of Sciences at the end of the seventies and used until now as frequently as the former one) are studied for more than twenty years [1-5]. Basically 
the term applies to semiconducting solid solutions in which one of cation components is substituted by a magnetically active species. Originally, the name denoted mostly II-VI compounds with a fraction of cations substituted by manganese. Later, the number of compounds included into the DMS family has expanded and now it encompasses a wide number of compounds: II-VIs, III-Vs, IV-VIs, II-Vs, etc., with many magnetic cations introduced into their respective lattices: $\mathrm{Mn}$, Co, Cr, V, Fe, Eu, etc. Some very early work has been done in Warsaw and its description can be found in the reviews quoted above. Initial motivation for the early interest was varied: it originated primarily in an intention to study an interaction of magnetic and "semiconducting" properties of materials, but it stemmed also from an extraordinarily high electron mobility observed in HgMnTe by Morrissy [6] and then, more specifically, from, has later become known as the giant spin splitting, observed in CdMnTe [7, 8] and ZnMnTe [9]. Apart from these motivations, in the course of investigations undertaken, there appeared a spectrum of interesting phenomena that singled out diluted magnetic semiconductors as a distinct and unique group of materials. The most interesting feature of the physics of DMS arises, of course, from the interaction between conduction and/or valence electrons with the magnetic component via the spin-dependent $s p-d$ interaction. The literature devoted to these materials is vast and covers many subjects ranging from their magnetic properties, lattice properties, electron transport properties, and optical and magnetooptical properties. At the beginning of the eighties, nearly parallel with birth of DMS physics, the semiconductor research worldwide became fascinated, and subsequently dominated, by studies of low-dimensional structures such as quantum wells and superlattices (to prove this statement one can have a look into proceedings of the School held in Nîmes in 1979 [10] where one of the first comprehensive papers on DMS was published by R.R. Gatązka and the present author and it appeared nearly back-to-back with the seminal article of L. Esaki on semiconductor superlattices). Already in the mid-eighties an effort was made to bridge the gap between these two branches of semiconductor physics [11-14] and first ideas appeared of low-dimensional structures involving components made of DMS. More importantly, in the same period of time first attempts to grow such structures by molecular beam epitaxy were undertaken, mostly in the United States $[15,16]$. These efforts were closely connected to an, now mostly abandoned, effort of constructing workable blue emitting laser made of II-VI materials with the magnetic aspect of the physics playing a less important role in this program [17-19].

This paper is not an ordinary review paper. Its aim is to describe a development of low-dimensional DMS program in the Institute of Physics of the Polish Academy of Sciences in Warsaw. Even with this disclaimer there are noticeable omissions in the paper. They are made because of the lack of space as well as because of lack of experience of the author in these specialized fields. These omissions include, for instance, structures of III-V ferromagnetic DMS which recently have 
attracted considerable attention in view of the prospect of their applications in future spintronics or even more remote quantum computations [20]. Similarly, no coverage is made of a fascinating subject of magnetic interlayer coupling in superlattices consisting of II-VI or of IV-VI constituents [21-23]. In other words, the paper concentrates on II-VI-based diluted magnetic semiconductor low-dimensional quantum structures fabricated and studied mostly in Warsaw.

Most of the low-dimensional systems involving DMS are now prepared with the use of molecular beam epitaxy [24]. However, the earliest reports were devoted to properties of quasi-two-dimensional electron gas in such systems that were formed either in metal-insulator-semiconductor (MIS) devices [25] — see Fig. 1 - or naturally occurring at the grain boundaries existing in crystals grown by the Bridgman method. In the former case a thin insulator deposited on the

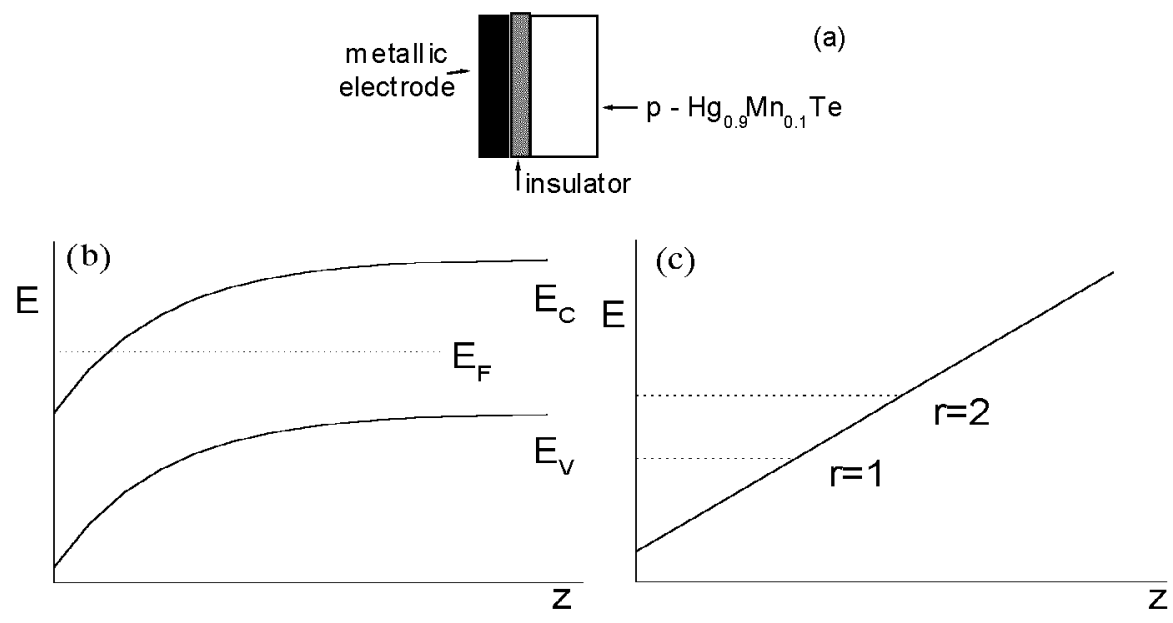

Fig. 1. (a) Schematic representation of a metal-insulator-semiconductor (semiconductor, in this example, being $p$-type diluted magnetic semiconductor $\mathrm{Hg}_{1-x} \mathrm{Mn}_{x} \mathrm{Te}$, as in [25]) structure; (b) the conduction $\left(E_{c}\right)$ and valence $\left(E_{v}\right)$ band edge profiles in MIS structure showing a band bending in the surface region and formation of the $n$-type inversion region with the Fermi level $E_{\mathrm{F}}$ located above the conduction band edge; (c) two lowest electron levels (edges of electric subbands) quantized by spatial confinement in an effective potential well with approximately triangular profile in the inversion layer.

surface of as grown, naturally $p$-type $\mathrm{Hg}_{1-x} \mathrm{Mn}_{x} \mathrm{Te}$ (with $\mathrm{Mn}$ content corresponding to a small but open-gap ordering of the $\Gamma_{6}$ conduction and the $\Gamma_{8}$ valence band edges). As an insulator a thin Mylar film or a layer of a special lacquer were used, on top of which a metallic gate electrode was further deposited (see Fig. 1a). The structures made with the Mylar film proved to be difficult to fabricate in a controlled and repeatable way. As in all MIS devices, a gate voltage of a proper polarity (attracting electrons to the gate) leads to an inversion of the conductivity of the charge carriers at the surface of the semiconductor by bending the bands 
in such a way that the Fermi level was shifted below the conduction band edge (see Fig. 1b). Under such conditions an asymmetric, approximately triangular potential well is formed which can quantize spatially the motion of electrons in the $z$-direction (taken as the direction perpendicular to the surface of the semiconductor). Solutions of a corresponding one-dimensional Schrödinger equation can be expressed in terms of the Airy functions [26] and the corresponding eigenenergies are known as the electric subbands. The presence of Mn ions in MIS devices made of DMS materials leads to a strong modification of the spin splitting characteristic of DMS (see below). These features were clearly observed in experiments on Shubnikov-de Haas oscillations of the conductivity in [27] in their MIS structures. Notorious instabilities present in those structures (both during their preparation and during their operation) made studies of the two-dimensional electron gas accumulated at grain boundaries particularly attractive. The origin of the spatial confinement of the carriers in the vicinity of the grain boundaries was probably due to sheets of charge impurities decorating these boundaries. These charges gave rise to a, this time symmetric, in approximation, triangular potential. The resultant quasi-two-dimensional electron gas possessed a superior mobility compared to that in MIS devices made of DMS and, therefore, the Shubnikov-de Haas experiments were particularly clean and meaningful in the former systems. The disadvantage of the structures with grain boundaries was that it was hardly possible to vary the concentration of the quasi-two-dimensional electron gas and, therefore, influence the occupation of the electric subbands. Also contacting these regions electrically in a reliable way was not an easy task.

For these reasons it became desirable to develop methods of fabrication of thin film structures made of DMS and their subsequent doping (preferably modulation doping) by one of the growth methods that already were known to be successful in producing good quality quantum wells and superlattice structures of various semiconductors, i.e., either by molecular beam epitaxy (MBE) or metalorganic chemical vapor deposition (MOCVD). The first method was preferable because of its greater versatility (the question of a throughput was not a deciding issue at the initial stage of research). With application of the MBE method various structures of II-VI diluted magnetic semiconductors soon became available (for references on early research on quantum structures of DMS see, e.g. [11]) and extensively studied. It quickly became evident that this particular growth method can produce materials otherwise not possible to achieve by other techniques, e.g., cubic MnTe (as opposed to "normal" MnTe with NiAs crystal structure) or cubic CdSe (which normally crystallized in the wurtzite structure). Also the efficiency of doping, both $n$-type and $p$-type, could be achieved to up-to-then unprecedented levels [28, 29]. More importantly, it became clear that incorporation of DMS materials into low-dimensional structure could lead to a wealth of new phenomena interesting from both basics physics point of view and their potential for applications $[12,14]$. 
In the Institute of Physics of the Polish Academy of Sciences a radical change took place only in 1993 where a small MBE system (model 620 made by, then, EPI) was installed. The system has six effusion cell ports (at present also the central view port, originally intended for pyrometric temperature measurements, is also used as a material source port accommodating a special cell equipped with its own internal shutter). On the other hand, it is possible to load up to six molyblocks at one time (see Fig. 2), thus shortening the time necessary for initial pump down, and considerably speeding up the work. The system initially configured for deposition of CdTe, ZnTe, MnTe (and, of course, their ternary and quaternary compounds) has been now used to grow also MgTe-containing materials [30], experiments were made with $\mathrm{Cr}$ as the magnetically active ingredient [31], as well as extensive studies of the doping of these materials with indium and iodine (for $n$-type) and, more recently with nitrogen (for $p$-type conduction). In particular, modulation doping of CdTe/CdMgTe quantum wells resulted in structures with two-dimensional electron gas displaying the highest mobility achieved so far. With modifications introduced over the years the system was successfully used for growth of so-called digital alloys and unique and very useful wedge- or step-structures [32].

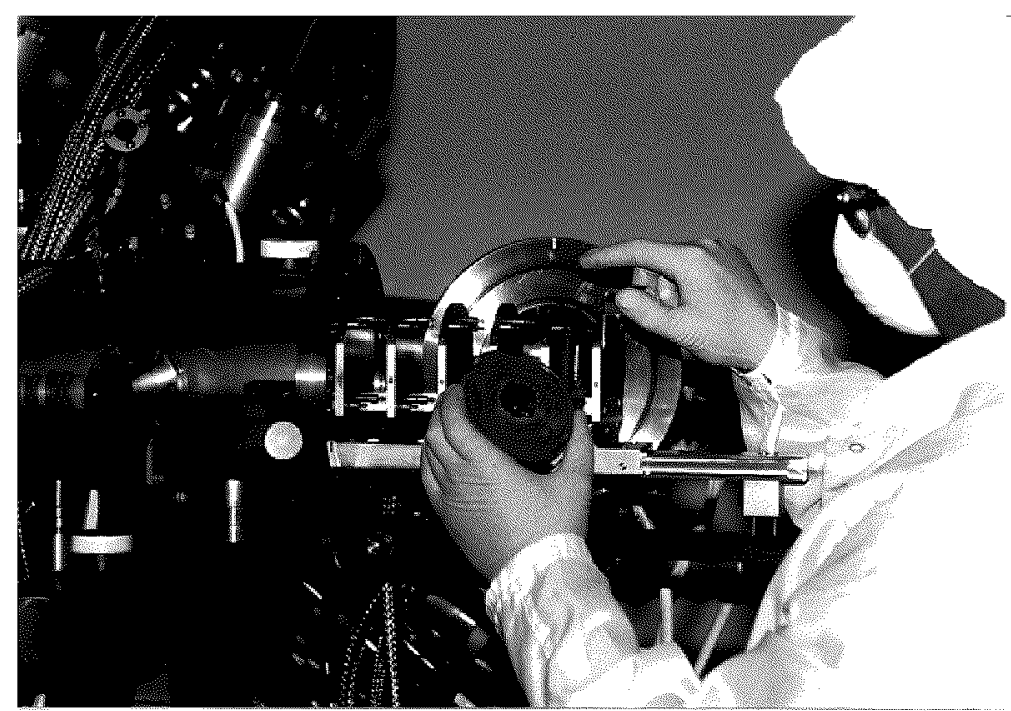

Fig. 2. Loading a molyblock with a piece of GaAs substrate to the introductory chamber of EPI 620 MBE system.

In the following text, after introducing some basic information about diluted magnetic semiconductors, we will mention some of the work done in the Institute of Physics stressing which of the initially dreamed of properties of DMS low-dimensional quantum structures were realized in fact, and which were either disproved or still remain in the realm of speculations. 


\section{Basics of diluted magnetic semiconductors}

The archetypal DMS material, $\mathrm{Cd}_{1-x} \mathrm{Mn}_{x} \mathrm{Te}$, increases its band gap at the $\Gamma$ point of the Brillouin zone upon addition of substitutional Mn nearly linearly with the molar fraction $x$ from its value of about $1.6 \mathrm{eV}$ in CdTe at helium temperatures to about $3.2 \mathrm{eV}$ in cubic MnTe (at the early stages called "hypothetical" material, see later in this paper). Inclusion of Mn does not only result in this effect. The most distinct feature is appearance of a strong $s-d$ and $p-d$ interaction between the conduction and, respectively, valence electrons with those strongly localized on the half-filled shell of substitutional Mn ions. This interaction is spin-dependent and makes itself particularly clearly observable upon application of an external magnetic field. In a nutshell, it leads to a modification (usually, a strong enhancement) of the spin splitting. The $g$-factors describing this enhanced splitting acquire an effective additional term which can be shown, in the mean field and virtual crystal approximations, to be proportional to the macroscopic magnetization appearing due to polarization of magnetic moments localized on Mn sites

$$
g_{\mathrm{eff}}=g^{*}+J_{s p-d} \frac{M}{\mu_{\mathrm{B}} B},
$$

where $g^{*}$ stands for the usual $g$-factor of either electrons or holes (originating, primarily, in the spin-orbit interaction in the host material), $M$ is the magnetization, $B$ - an external magnetic field, $\mu_{\mathrm{B}}$ being the Bohr magneton and $J_{s p-d}$ - the interaction proportionality constant (traditionally denoted by $\alpha$ in the case of $s-d$ interaction involving conduction electrons, and by $\beta-$ in the case of $p-d$ interaction involving valence band holes). The magnitude of $J_{s p-d}$ is such in the materials in question that the second term in Eq. (1) can easily be greater than the first one. It depends on the magnetic field and on the temperature that both determine the magnetization. In crystals where the inter-Mn exchange interactions is significant, i.e., where the molar fraction $x$ is large enough for pairs and larger Mn clusters to be probable to occur, these interactions (which are antiferromagnetic II-VI DMSs) can reduce the magnitude of the magnetization. In other words, the mutual cancellation of the magnetic moments in an interacting pair of nearest neighboring pairs can win over an increased density of the magnetic moments. As a result the $s p-d$ exchange contribution to the electron and/or hole $g$-factor can be also reduced. "Optimum" conditions (i.e., corresponding to the maximum of the magnetization at a given temperature and a given magnetic field) can be usually found in a neighborhood of $x=0.1-0.15$.

Let us stress here that in order for the exchange contribution to the spin splitting of the conduction electrons and of the valence band holes in II-VI diluted magnetic semiconductors it is necessary to apply an external magnetic field. Usually low temperatures also are equally vital since the magnetization at elevated temperature conditions is negligibly small. It is related to the fact that these substances are either paramagnetic (for low values of the molar fraction $x$ ) or form, at 
sufficiently low temperatures, a spin-glassy or antiferromagnetic state - for higher values of $x$ - for which the macroscopic magnetization vanishes in the absence of the field. This is a considerable drawback of these materials if one has in mind their applications in realistic devices since the need of applying the magnetic field complicates the design of, say, spin filters, and also since room temperature operation of such devices is a natural prerequisite. From this point of view III-V based diluted magnetic semiconductors, which are ferromagnetic at high temperatures [20], seem at present to be more promising, however, the oxide semiconductors, in particular $\mathrm{ZnO}$ with $\mathrm{Mn}$ and specially with $\mathrm{Co}$, are equally intensively studied in this context (as evidenced by a number of contributions at a recent international conference on II-VI compounds in Bremen; proceedings to appear in [33]). With all these reservations, DMS made of II-VI compounds containing manganese remain an important testing ground for new ideas and designs.

The existence of the $s p-d$ interactions in DMS materials manifest themselves not only as an increased spin splitting in the presence of a magnetic field. One of the most important consequences of the $s p-d$ interaction, when going beyond the mean field approximation, is a possibility of formation of magnetic polarons, i.e., self-localized states of a carrier and surrounding cloud of magnetization [34-37]. Bound (either by donors or acceptors) magnetic polarons were unambiguously evidenced in three-dimensional DMS samples as, e.g., zero-field splitting observed in the Raman scattering experiment [38-40]. It turns out that of utmost importance for the formation and stability of magnetic polarons are thermal fluctuations of the magnetization which can be particularly large in view of a finite volume that the polaron occupies. It is evident, therefore, that the reduction of the dimensionality of the quantum structure (via the spatial confinement of the carriers) can have a profound effect on stabilization of the magnetic polarons [41, 42]. Parenthetically, the same fluctuations of the spin degree of freedom can contribute also to the modifications of the fundamental band gap and, particularly, to its temperature dependence, however, this contribution is less pronounced since it is basically a higher order perturbation effect [43].

The dependence of the magnetic polaron binding energy on the dimensionality gave rise to hopes of a possibility of observation of free magnetic polarons in low-dimensional structures [44] (it was known that free magnetic polarons are unstable in realistic three-dimensional DMS). Soon, however, it became clear that the magnetic polarons whose clear optical signatures were found in DMS quantum wells [45] were due to excitons that were not completely free but additionally stabilized by fluctuations of the quantum well width. Nevertheless, the binding energy of magnetic polarons was found to depend sensitively on the width of the quantum well [46] (and, thus, on the effective dimensionality of the system). In particular, in quasi-zero-dimensional quantum dots there are reports of this energy acquiring very large values [47]. 
Large spin splitting of DMS materials is the source of a giant Faraday rotation effect $[48,40]$. Therefore, one of the applications of DMS materials was to use them as optical isolators in optical circuitry [49]. Unfortunately, particularly large Faraday rotation angles are observed in the spectral region which is inherently associated with a strong absorption of light. This fact makes it difficult to find a reasonable trade-off conditions between the attenuation and effectiveness of the isolator. The Faraday rotation, on the other hand, is expected to be even stronger in the two-dimensional configuration [50]. However, the difficulty mentioned above remains equally restrictive and as shown in [51] the hopes of making useful Faraday isolators of quasi-two-dimensional DMS quantum structures remain problematic.

The unique character of DMS materials as building blocks of quantum structures is most evident when comparing the typical values of the valence and conduction band offset in semiconductor heterostructures with possible to achieve values of the spin splitting described by Eq. (1). It turns out that under experimental conditions easily available in laboratories these two quantities can have comparable magnitudes with the spin splitting sometimes exceeding the band gap discontinuity. Of course, as mentioned, such a situation does require application of an external magnetic field (see Fig. 3).

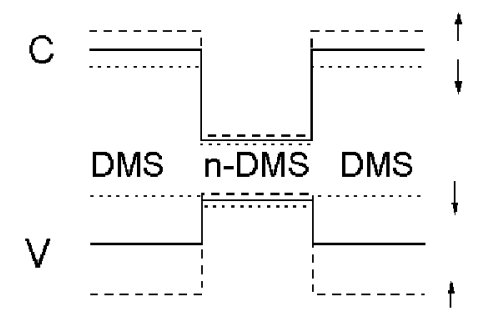

Fig. 3. Conduction (C) and valence (V) band edges in a quantum structure made of diluted magnetic semiconductor (DMS) barriers, e.g., $\mathrm{Cd}_{1-x} \mathrm{Mn}_{x} \mathrm{Te}$, and non-magnetic (n-DMS) quantum well, e.g., CdTe, in the absence (solid line) and in the presence (dotted and broken lines) for, respectively, spin down and spin up states.

This inherent feature of quantum structures made of DMS materials made von Ortenberg [52] put forward an idea of a spin superlattice. Starting with a superlattice made of non-DMS and DMS sequence of layers ( $\mathrm{HgSe}$ and $\mathrm{HgMnSe}$, in the original proposal of von Ortenberg), whose band edges in the absence of the fields would be located at the same energy, and then, by applying a magnetic field, one arrives at a situation where for a given spin direction the carriers experience either a potential maximum in the DMS layer and the potential minimum (quantum well) in the non-DMS layers (for the opposite spin direction the maximum and the minimum would occur in, respectively, non-DMS and DMS layers). As a result one could spatially separate carriers having opposite spins. The idea proved to be not easy to realize. Only after some ten years there were two unambiguous reports 
on observation of such spatial separations of spins. This was achieved, however not in mercury-based narrow gap materials of the original proposal but in wide gap zinc compounds [53-55].

Closely related to the idea of a spin superlattice was the notion of type-I-to-type-II transition induced by a magnetic field [56]. In the literature on quantum well structures, the type-I structure denotes a quantum well design where both types of carriers (electrons and holes) are localized in one spatial region of a heterostructure. Conversely, type-II heterostructure denotes a sequence of layers resulting in the hole localization in regions which constitute barriers for the conduction electrons. Since the spin splitting in DMS materials can exceed the (usually small) valence band offset, a situation is conceivable (for instance in $\mathrm{CdTe} / \mathrm{CdMnTe}$ heterostructures) that the type-I design in zero magnetic field becomes (for one spin direction of holes) a type-II configuration with associated sudden decrease in, e.g., photoluminescence intensity induced by a sudden field-induced reduction of an overlap of the wave function of electron and holes. Unambiguous experimental evidence of this transition also proved not to be an easy task but, finally, it was shown that such a transition does take place $[57,58]$.

The experimental proofs of both ideas, the superlattice and type-I-to-type-II transition, relied on careful measurements of the rate of the field-induced shift of the energy of light either emitted (in photoluminescence experiments) or absorbed (in transmission experiments) in spatially separate regions of a heterostructure in two opposite circular polarizations. These achievements, in turn, gave a basis for a concept of spin function mapping [59-61]. Knowing the position of a thin magnetic layer in the structure one could measure experimentally in a quantitative way the probability density of finding a carrier in these areas by probing the spin splitting of excitons in those structures. The magnetic markers in these particular structures were very thin (often below one monolayer) so that the mapping in question could be very precise indeed.

Another useful application of the large spin-splitting feature in DMS heterostructures was a quantitative characterization of quality of the interfaces between the quantum well and quantum barrier regions. For the lack of space we refer here the reader to comprehensive papers summarizing the results of this series of investigations $[62,63]$.

While the above examples might serve as cases of "success" stories there are a number of predictions (or speculations, as some prefer to call them) that remain to be either realized or were outright disproved [12]. One of those ideas still to be pursued is illustrated in Fig. 4 where a structure resembling what is now known as a cascade laser structure is depicted. The use of diluted magnetic semiconductor layer as a component makes, in principle, in such structures that a tunneling between successive quantum wells is spin selective with a consequence being that the light emitted from such a structure is circularly polarized. Let us remark in 


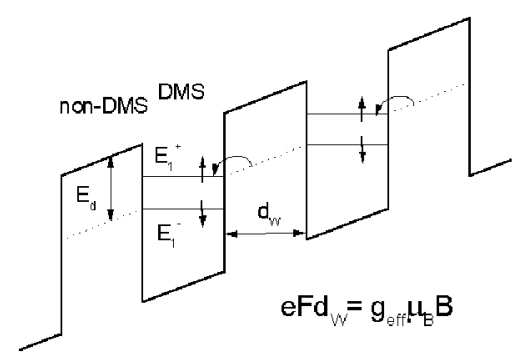

Fig. 4. The conduction band edge profile of a quantum structure with DMS quantum wells, e.g., $\mathrm{Cd}_{1-x} \mathrm{Mn}_{x} \mathrm{Te}$, and non-magnetic barriers, e.g., $\mathrm{Cd}_{1-y} \mathrm{Mg}_{y} \mathrm{Te}, x<y$, subject to external electric and magnetic fields parallel to the growth direction. Two spin-split confined electron states are shown in the quantum wells, together with the shallow donor state (which shows only very small spin splitting, here neglected altogether) in the barriers. By choosing the quantum well width and that of the barriers one can satisfy the relative location of the levels as shown in the figure, enabling series of cascade-like spin-flip transitions.

passing that the concept of spin-dependent tunneling (for an early experiments see, e.g. [64]) has acquired new importance in the concept of spin injection (see below).

One final remark that one cannot avoid to make: the speculations or predictions of interesting new features often made use of narrow gap DMS involving mercury compounds. These turned out to be difficult to obtain and equally difficult to interpret. This fact was partially at least connected to an enormous effort that was put in development of growth techniques of wide band gap II-VI materials that were to be the materials for blue lasers, with the effort spent in the area of narrow gap materials being somewhat less extensive. One can only hope that nowadays a certain balance is restored [65].

After this introductory part, the rest of the paper very briefly presents the main directions of the diluted magnetic semiconductor related research carried out over the period of nearly eight years of activity of the Laboratory of Growth and Physics of Low-Dimensional Crystals in the Institute of Physics of the Polish Academy of Sciences in Warsaw.

\section{Beyond the natural symmetry}

Epitaxial growth techniques by an appropriate selection of substrate materials and a careful choice of the growth conditions (substrate temperature and chemical composition of the impinging molecular beams in the case of MBE) enable fabrications of crystals that are otherwise impossible to be obtained by alternative equilibrium growth techniques. In particular, it is possible to impose the crystal symmetry of a substrate on a film that grows on its surface. One of the examples 
of such possibilities was successful growth of CdSe in the zinc blende form [66] that was fundamentally crucial to numerous works that followed aiming at obtaining selenide-based structures for laser diodes emitting in the blue range of the spectrum. Prior to this achievement the prevailing opinion was skeptical as far as use of the selenides (which naturally crystallize in the hexagonal wurtzite form) was concerned - they seemed to be incompatible with GaAs substrates that were considered as the substrates of choice at that time (and they remained such in many instances until today).

Cubic, zinc blende form of MnTe is another example of such symmetry of the cubic GaAs substrate being imposed on the grown film [67-69]. The interest in this particular material was further motivated by the fact that if grown successfully it would represent one of the very few examples of cubic antiferromagnetically coupled systems with first nearest neighbor interactions prevailing by approximately an order of magnitude over those between the next nearest magnetic neighbors. Thus it would provide an experimental material to test the predictions of Anderson [70]. In fact, a whole series of samples were grown by $\mathrm{MBE}$ of $\mathrm{Cd}_{1-x} \mathrm{Mn}_{x} \mathrm{Te}$ with $x$ exceeding 0.66 (which was the limit achieved by other growth techniques in the case of bulk crystals) and covering the entire range from $x=0$ to $x=1$. These samples were carefully studied from their magnetic properties point of view $[71,72]$ and showed that a well defined border line between antiferomagnetically ordered phase and the spin-glassy behaving phase (with its characteristic remanent magnetization properties) is very difficult to pinpoint, if it exists at all. These very samples were also used in studies directed to observation of magnons, both by the Raman scattering and neutron scattering techniques [73, 74]. As a spin-off of these studies, it turned out that the predictions that cubic MnTe films were structurally unstable and, after certain critical thickness was exceeded turned to their "natural" hexagonal NiAs form, were not substantiated. It was possible to grow cubic MnTe films as thick as $8 \mu \mathrm{m}$ with the patience of the MBE system operator being the only serious limitation. Studies of structural and optical properties of this material proved that the extrapolated values of the crystal parameter and energy gap of zinc blende MnTe were fairly accurate and did not correspond to a merely "hypothetical", but quite real, substance.

Growth of cubic $\mathrm{MgTe}$ and $\mathrm{MgTe}$-rich $\mathrm{Cd}_{1-x} \mathrm{Mg}_{x}$ Te ternaries represented yet another problem. Above, approximately, $x=0.6$ this compound is highly hygroscopic and decomposes quickly in air. Thus some sort of protective layer were necessary in order to measure, e.g., the lattice parameter of this compound series $[75]$.

Molecular beam epitaxy was also crucial (although less successful) in extending the solubility limits of transition metal ions other than $\mathrm{Mn}$ in $\mathrm{CdTe}$ or ZnTe host matrices. In particular, it was possible to obtain by this method crystals containing up to $x=0.03$ of $\mathrm{Cr}$ with good optical quality that made possible examination of their reflectivity properties possible [31]. 


\section{Digital growth and parabolic quantum wells}

The idea of "digital" growth of ternary alloys [76] proved itself to be useful on several distinct occasions. In particular, a precise profiling of a shape of the confining potential of a quantum well proved to be possible also in the case of II-VI materials including DMSs. In fact, growth of parabolic, trapezoidal, triangular, and half-parabolic potential has been achieved [77]. The growth of those structures not only demonstrated skills of MBE operators but made possible determination of the band offsets between $\mathrm{CdTe}$ and MnTe as well as between CdTe and MgTe with accuracy that could not be achieved otherwise [78]. In those studies use of asymmetric half-parabolic quantum well turned out to be particularly helpful since lack of the mirror-reflection symmetry of the confining potential in the plane perpendicular to the growth direction lifted some restrictions of the selection rules. As a result more characteristic lines became observable in the optical spectra which put a more stringent limitations to the errors ensuing in the subsequent analysis of these spectra.

The other example where digital type of growth was employed is an attempt to grow an ordered alloy of $\mathrm{Cd}_{1-x} \mathrm{Mn}_{x} \mathrm{Te}$ on $\mathrm{GaAs}$ substrate whose orientation differed from the typical (001) orientation and actually was parallel to (120) direction. As predicted by Wolff and Ram-Mohan [79] if three CdTe and one MnTe monolayers were deposited consecutively along such a crystallographic direction, the resulting ordered alloy of $\mathrm{Cd}_{0.75} \mathrm{Mn}_{0.25} \mathrm{Te}$ would have no nearest $\mathrm{Mn}-\mathrm{Mn}$ neighbors (only second nearest neighbors being possible whose antiferromagnetic interaction is by about an order of magnitude weaker than that between the nearest neighbors). In consequence, it was expected that the effective magnetization of such an ordered alloy would be considerably greater than that in the disordered alloy having the same Mn content. Further, such greater magnetization would correspond to a greater spin splitting as a consequence of Eq. (1). Growth of such structures was proved to be possible [80] but, surprisingly, no enhancement of the spin splitting of the excitonic states was observed. On the other hand, these alloys were distinctly different from normal disordered counterparts because the binding energy of magnetic polarons measured in those samples were different than those on twin samples grown along (100) direction. The latter difference was explainable in terms of the anisotropy of the hole effective mass [80]. The exact reason for the failure to observe increased spin splitting in these (120)-oriented alloys remains not clear.

Also attempts to increase the effectiveness of $n$-type doping using In dopants of CdMnTe and CdMgTe ternary compounds by employing digital mode of growth proved not to be very successful. This failure had certainly to do with the fact that CdTe (which was relatively easier to dope with In) possesses a smaller band gap than the ternaries with $\mathrm{Mn}$ and $\mathrm{Mg}$. In the latter compounds, in particular, the metastable effects and DX-like behavior of the In impurity was, therefore, observed [81, 82]. It is worth noting that the above mentioned trick seems to 
be working in the case of GaSb/MnSn structures [83] owing to a peculiar band offsets between these two materials. The latter result gives hopes that such digital materials will be important in the future spintronic materials. On the other hand, changing the dopant species to iodine resulted in a possibility of doping in the barrier regions of, say, CdMgTe/CdTe quantum structures. Such modulation doped structures were found to lead to two-dimensional electron gas with extraordinarily high mobility [84] enabling observation of very clear integer quantum Hall effect (with a pronounced spin split Hall plateaus in the case of CdMnTe/CdMgTe structures [84]) but also intriguing optical anomalies in the region corresponding to fractional Hall effect $[85,86]$. Parenthetically, doping with nitrogen which results in p-type conductivity in ZnTe and CdTe, has been recently commenced in Warsaw. The first result (shown in Fig. 5) indicate that a good quality two-dimensional hole gas might be achievable also in II-VI materials, possibly including also DMS materials.

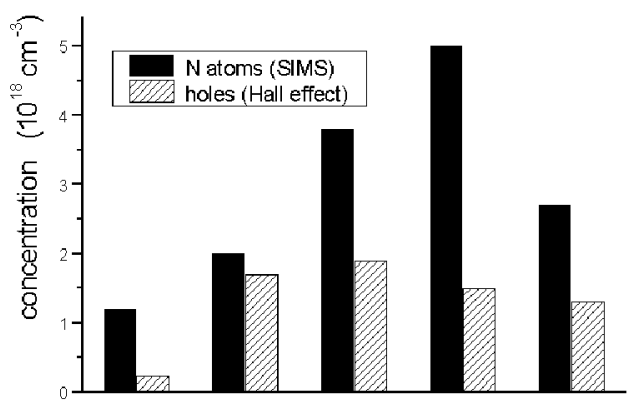

Fig. 5. Effectiveness of $p$-type doping of MBE-grown ZnTe with nitrogen from rf-plasma source in several samples. The black bars indicate amount of nitrogen detected by secondary ion mass spectroscopy (SIMS) measurements and the hatched bars - the concentration of holes measured at room temperature by the Hall effect.

The above examples of digital mode of growth do not exhaust the cases reported in the literature. For instance, samples used for the wave function mapping mentioned above [61] were prepared by this very method, similarly to samples used in studies of Crooker et al. [87] and studies of initial stages of growth of self-assembled quantum dots [88].

\section{Tunable $g$-factor}

As mentioned, the enhancement of the hole or electron $g$-factor is an outstanding feature of diluted magnetic materials. This feature can be used to advantage when studying phenomena that crucially depend on the spin polarization of the carrier gas. One of the examples of such use of diluted magnetic semiconductors was a recent study of formation of negatively charged excitons, known also 
as trions [89]. By a careful control of the composition of $\mathrm{Cd}_{1-x} \mathrm{Mn}_{x}$ Te layers it was possible to obtain samples that changed the sign of their electron $g$-factor as the magnetic field was swept, as shown in Fig. 6. Because the sign of the first and the second term in Eq. (1) happen to be opposite in the case of the conduction electrons in $\mathrm{Cd}_{1-x} \mathrm{Mn}_{x}$ Te it is possible that for a certain value of a magnetic field (appearing in the denominator of Eq. (1)) there is a complete cancellation of each other. The value of this particular magnetic field does depend on the magnetization, i.e., on the temperature and Mn molar fraction.

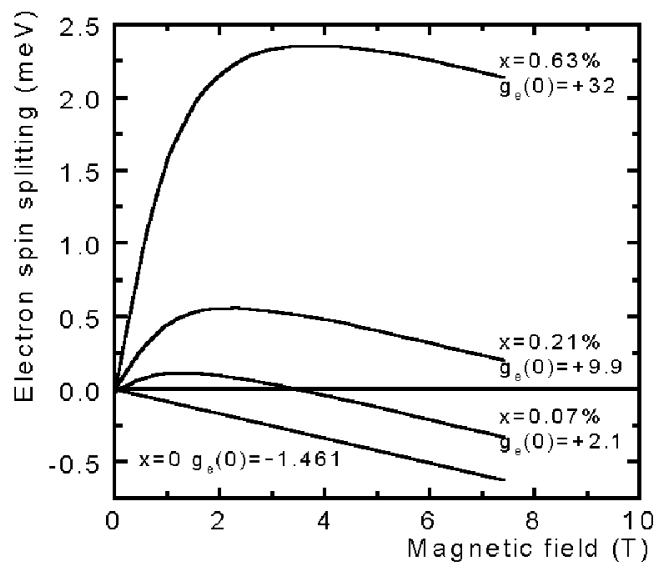

Fig. 6. The spin splitting of the conduction band states in $\mathrm{Cd}_{1-x} \mathrm{Mn}_{x}$ Te with various molar fractions $x$ measured at $T=1.8 \mathrm{~K}$ as a function of magnetic field. The effective $g$-factors at small fields, $g_{\mathrm{e}}(0)$, are dominated by the second term in Eq. (1) resulting in positive values of the spin splitting, with the exception of $x=0$, where only the first term is present leading to a negative value of the spin splitting [113].

A less trivial possibility of tuning the $g$-factor stems from its dependence on the dimensionality [90]. In the quantum wells the conduction electron $g$-factor does depend on the thickness of the quantum well. This dependence is associated with the fact that the $p$-like admixtures to electronic wave functions depend on the quantum well width through their eigenenergy. These admixtures, in turn, determine to what extent the kinetic exchange mechanism (strong, but forbidden by symmetry in the case of $s$-like wave functions) becomes effective and contributes to the resultant $J_{s p-d}$ constant. Again, since the sign of the exchange constant given by the direct exchange mechanism (and allowed in the case of purely $s$-like electrons) is opposite to that of the contribution produced by the hybridization-driven exchange, it is in principle possible for the electronic $g$-factor to vanish completely by carefully choosing the quantum well parameters. Even greater modifications of the effective exchange constants than in quantum wells are expected theoretically in the case of zero-dimensional quantum dots [91]. Indications that this is indeed the case were reported recently by Japanese researchers [47]. 
The studies that aim at an unambiguous proof that, e.g., $\alpha$ varies with the quantum well width are greatly facilitated if a set of samples is available that differ between themselves only in just one parameter, the width. This is only possible if the samples are grown in a single MBE process. Only then one can be sure that there no change of the growth parameters occurred, something that is impossible to achieve from one run to another. In fact, fabrication of such set of samples is possible [92] by making use of an ingenious method introduced by Wojtowicz, which involves growth of wedge-like or step-like samples in which only one parameter (for instance, the width of a quantum well layer) is varied along the length of a sample by moving (at a constant speed or in steps) a main shutter in front of a growing structure while the material is deposited.

\section{Spin injection and spin filters}

Recent achievements in injecting spin polarized carriers either from ferromagnetic semiconductor or through a DMS spin aligners [93, 94] to a LED region of a non-magnetic quantum well structures increased hopes in achieving a working spintronic devices. These works were preceded by a seminal work of Oestreich et al. [95] who made use of DMS aligner idea, however, in their case the spin polarized injection made use of optical excitation with a given circular polarization of light in the aligning CdMnTe layer followed by a diffusion of excitons to a non-magnetic part of the structure without a complete loss of the spin polarization. A natural question arose how critical was the quality of the interfaces between the DMS spin aligning layer and the non-magnetic part where the exciton (or electron-hole) recombination took place. In order to shed light on this issue, a structure was grown which on purpose exaggerated the density of lattice parameter mismatch-generated dislocations at the interface. The structure consisted of a GaAs/GaInAs quantum well structure grown in a separate run in a dedicated III-V MBE system, which was then transferred in air to the II-VI MBE system where after removal of the protective As layer it was regrown with a layer of $\mathrm{ZnMnTe}$ (see Fig. 7). As revealed by transmission electron microscopy cross-sectional images, the interface between the II-VI and III-V part of the structure although retaining a crystal character (no amorphous layer present) is the source of a great number of dislocations (Fig. 8). In spite of their presence, careful optical studies of the structure showed without doubt that the carriers (or excitons) that diffused to the non-magnetic quantum well region buried up to $300 \AA$ below the II-VI/III-V interface and there they recombined radiatively, did retain a degree of their spin polarization [96]. Similar study was carried out in parallel (and published earlier) in the selenide system $[97,98]$. Both experiments indicate that spin polarization of carriers is a very robust property and it survives a transport through an even very crystallographically imperfect regions of semiconducting material. It makes the theoretical calculations concerning the spin filtering device [99] less speculative and closer to reality. 


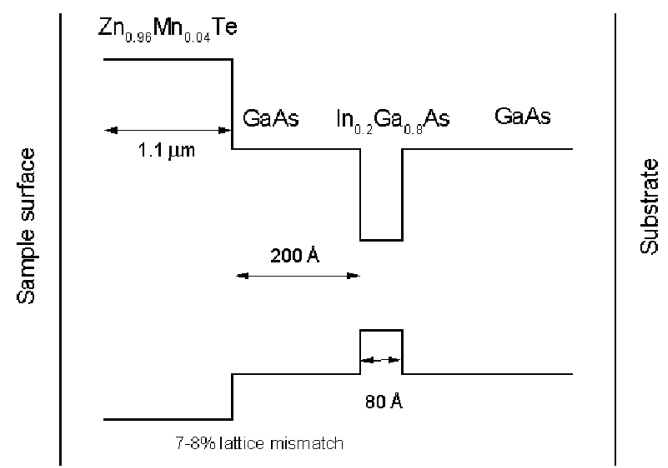

Fig. 7. Schematic representation of the quantum structure used in studies of spin injection across highly lattice-mismatched interface between the III-V detecting quantum structures and II-VI DMS spin aligner [96].

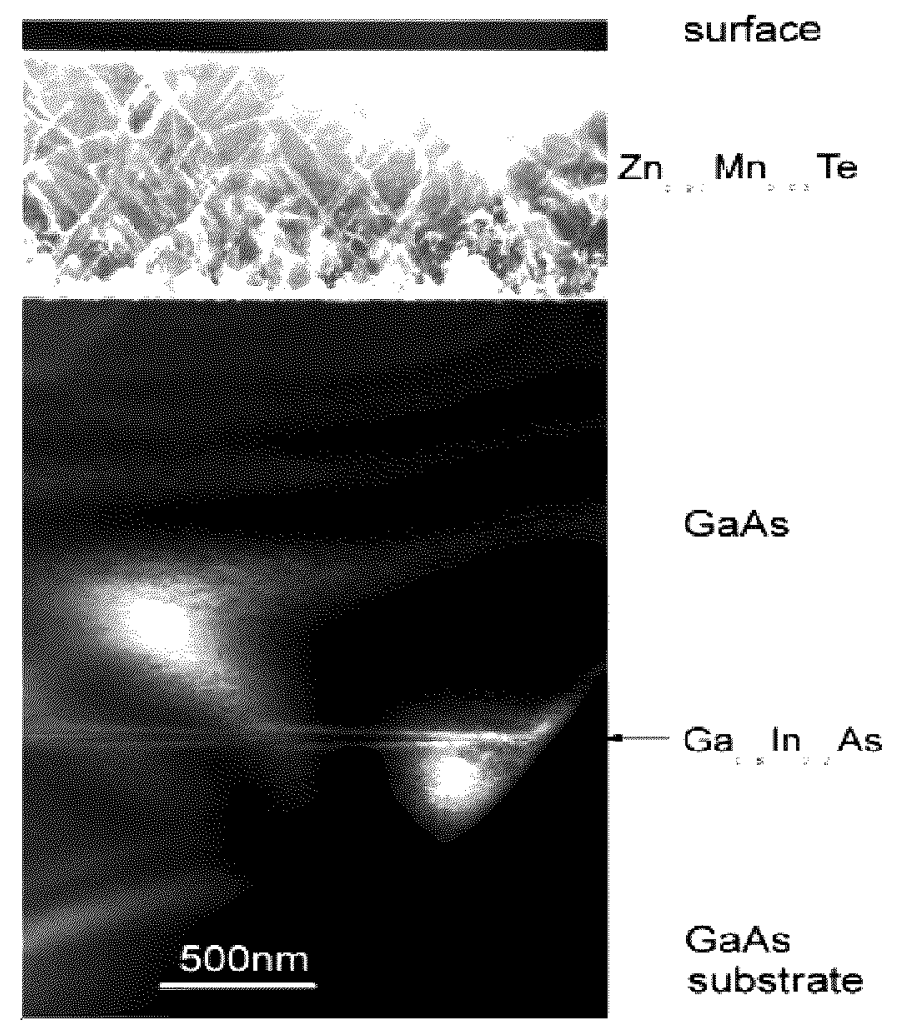

Fig. 8. Cross-sectional transmission electron micrograph of the structure shown schematically in Fig. 7, however, with the GaAs top layer being $2000 \AA$ wide. 


\section{Self-assembled quantum dots}

As in III-V heterostructure systems with large lattice mismatch (InAs/GaAs being the classic example) also II-VI grown by MBE can form self-assembled quantum dots. In the case of II-VI there is an agreement, however, that the exact machanism of growth responsible for the self assembly differs from the Stranski-Krastanow mechanism $[100,101]$. The dots in the telluride systems grown in Warsaw have a particularly large density which gives rise to a number of interesting cross-talk effects in the power and temperature dependence of their optical signatures [102]. This is also the reason that in the case of the tellurides it is very difficult, if possible at all, to register optical spectra corresponding to a single quantum dot, as exemplified in Fig. 9 where a whole series of very narrow lines corresponding to a luminescence of as many as 50 dots is visible in a microphotoluminescence ( $\mu$-PL) experiments performed through an aperture in a metallic mask with $150 \mathrm{~nm}$ in diameter [103]. In the case of the selenide counterparts the density of the dots is smaller and even conventional $\mu$-PL with the use of a microscope (spatial resolution of the order of $1-2 \mu \mathrm{m}$ ) can resolve the spikes corresponding to individual dots, as can be seen in Fig. 10a.

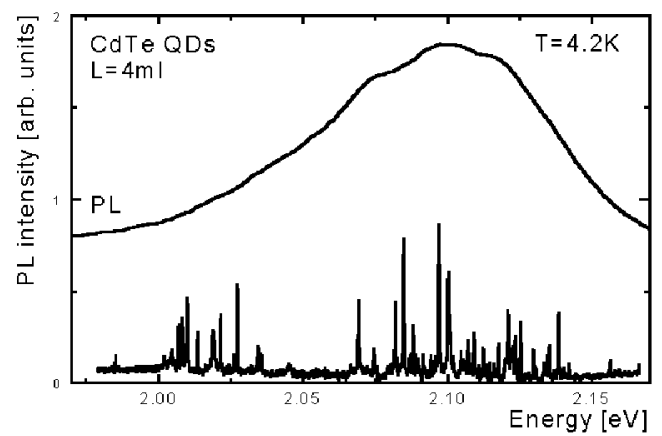

Fig. 9. Photoluminescence from a macroscopic region of a sample containing self-assembled CdTe quantum dots in ZnTe (top curve) and from a small region (defined by a small aperture $150 \mathrm{~nm}$ in a metallic mask) of the same sample (bottom curve). A series of sharp features is observed each corresponding to a single quantum dot. The nominal thickness of deposited CdTe layer forming quantum dots is 4 monolayers (ml).

It is interesting to note that that the average width of the individual lines does not depend on the exact chemical composition and/or size of the quantum dot (the dots are known to be interdiffused to a considerable extent). This is further supported by the $\mu$-PL spectra of samples where a ternary (CdMgSe) material was deposited when growing the layers that formed the self-assembled quantum dots (see Fig. 10b). The above statement remains true provided that the ternary material is non-magnetic. Even a small admixture of Mn ions during the self-assembly of the dot layer results in a complete disappearance of the fine 

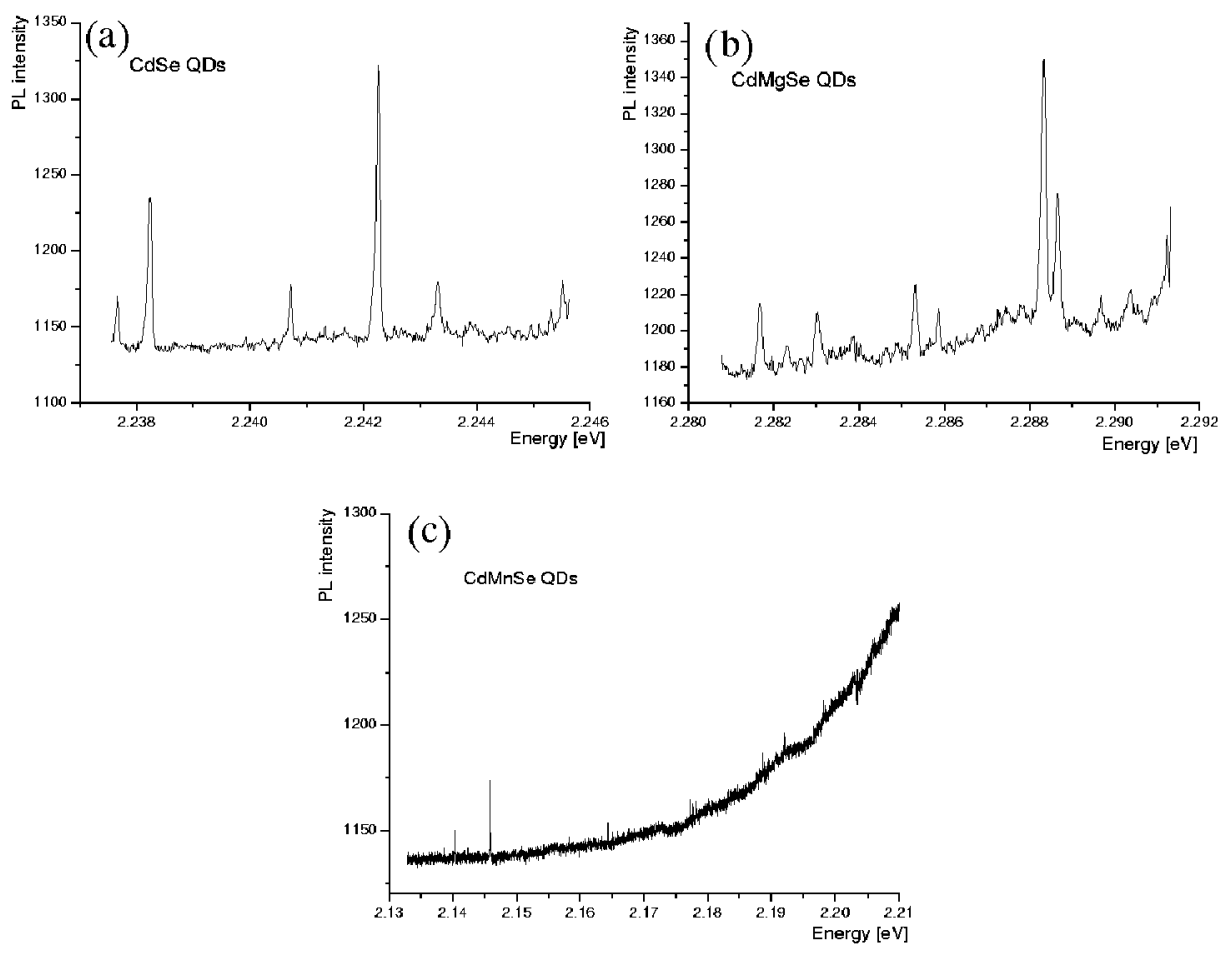

Fig. 10. Microluminescence of samples containing (a) nominally CdSe, (b) CdMgSe, and (c) CdMnSe self-assembled quantum dots embedded in ZnSe. The $\mu$-PL intensity is in the same arbitrary units in each part of the figure.

structure due to the luminescence of individual quantum dots. The small sharp structures seen in Fig. 10c are just noisy signals. This fact is confirmed further by measurements through small apertures in metallic masks both in the selenide system $[104,105]$ and telluride system $[106,103]$ where only very broad structures (broader by roughly an order of magnitude compared to the case of non-magnetic quantum dots) could be detected. The broadening is related to an additional source of fluctuations present in the magnetic system, namely the fluctuations of the magnetization in dots that are characterized by a finite small volume [105, 104]. Roughly the line width should follow the dependence that can be derived in a manner similar to that used in considerations related to the magnetic polaron [107] which predicts that it should be proportional to $\sqrt{\partial M / \partial B}$, where $M$ represents magnetization of the system and inversely proportional to $\sqrt{V}$, where $V$ represents the volume to which the polaron is confined (e.g., the volume of a quantum dot). The magnitude of the fluctuations of magnetization (and thus of the line width of individual quantum dot luminescence) does critically depend on the volume of the system. It is likely, therefore, to be of greater importance in the telluride system 
where the dots grown so far are more dense but also smaller than in the case of the selenides.

\section{Hybrid ferromagnet/DMS structures}

The fact that in II-VI DMS materials their interesting spin properties occur only when an external magnetic field is applied (which is related to their antiferromagnetic nature of $\mathrm{Mn}-\mathrm{Mn}$ interactions) represents a drawback when one intends to use them in working spintronic devices. Therefore, already two decades ago an idea of a hybrid structure was put forward [108]. In such structure one could make use of a magnetic field exerted by a metallic ferromagnet positioned very close (on an atomic scale) to a DMS layer or quantum structure, thus affecting the spin splitting in the latter. For instance, one could use such fringe fields to shape the band edges in such a fashion that an additional localization of the carriers in a two-dimensional quantum well is induced leading to formation of quantum wires or quantum dots. Recently, several attempts to prepare structures of such type have been performed. The experiments do indicate that the principle of such hybrid structures is realistic. A schematic view of a typical structure used in these experiments is shown in Fig. 11. It consists of a diluted magnetic semiconductor quantum well buried not very far away from the sample surface. Then, by a combination of lithoghaphic lift-off technique and low-pressure sputtering small rectangular islands of ferromagnetic substances were deposited on the surface (shown

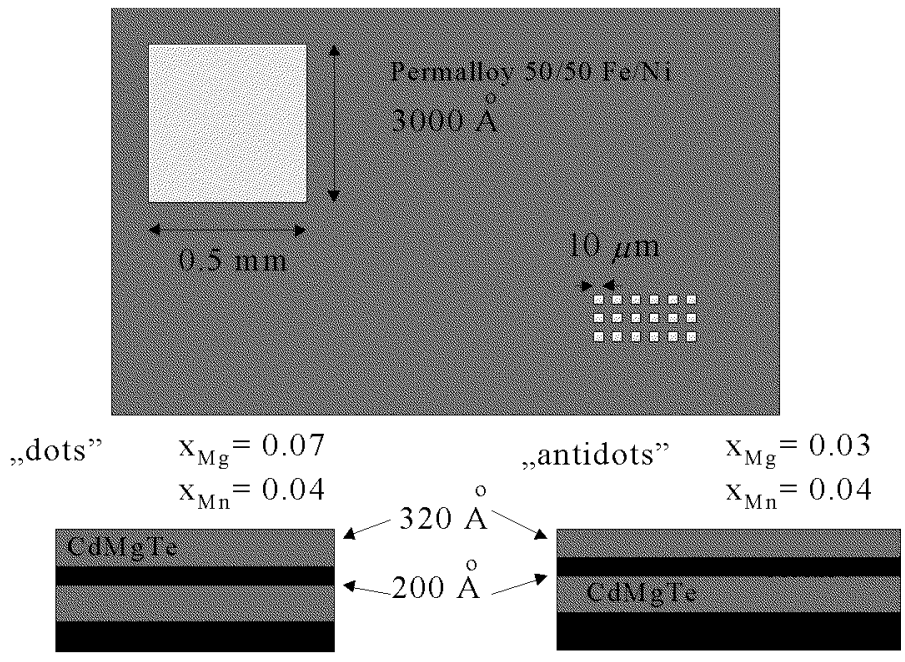

Fig. 11. Schematic representation of a typical hybrid structure used in studies of the effect of fringe fields of micromagnets made with permalloy. The upper part shows the top view, the lower part - the cross-section. 
(a)
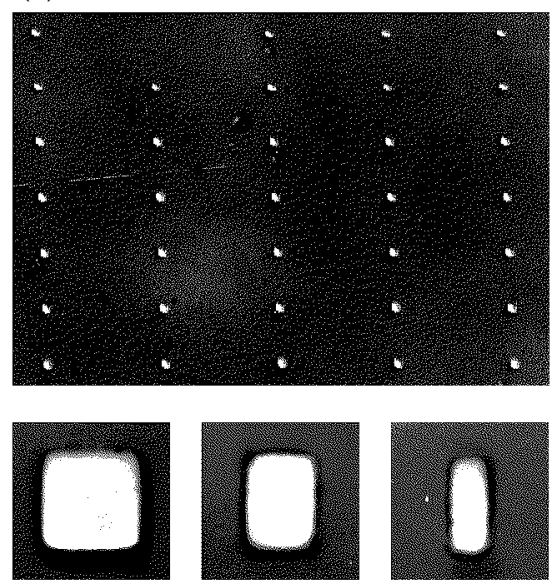
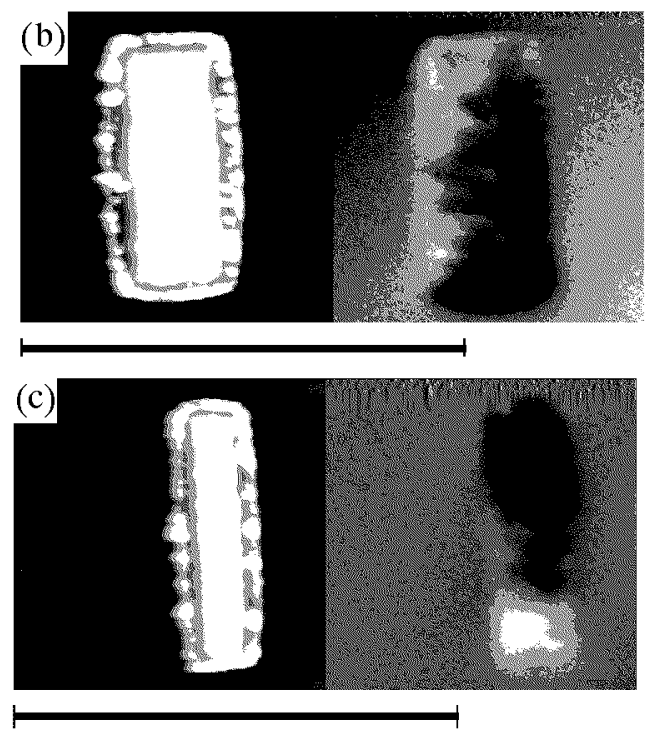

Fig. 12. (a) Nomarski contrast microscopic view of a hybrid structure with Fe micromagnets having thickness of $2000 \AA$ and lateral dimensions $6 \times 6,6 \times 4$, and $6 \times 2 \mu \mathrm{m}^{2}$ (see lower panels); (b) and (c) atomic force (left panels) and magnetic force (right panels) microscopy of these micromagnets showing imperfections of the edges (so-called, side walls) and clear, stable domain structure.

in Fig. 12a) with characteristic extensions in the range $2-10 \mu \mathrm{m}$. The sample with an array of ferromagnetic islands is then magnetized by placing it in a magnet in a field of several tesla. The experiments performed so far in Warsaw involved Fe, permalloy, Co, and Dy as the materials forming the micromagnets. Figures $12 \mathrm{a}$ and b show atomic force (AFM) and magnetic force microscopy (MFM) images of the magnets made of iron. While the AFM images clearly indicate that further work has to be done in order to remove the side walls, the MFM images show that it is possible to magnetize these magnets so that they have relatively simple domain structures (see, particularly Fig. 12b). The MFM images taken several days after the initial magnetization show that the domain structure is, in fact, quite stable. If a micromagnet with only one domain is achieved, the magnetic field component in the spatial region of the buried DMS quantum well due to the presence of such a magnet atop of the sample can be calculated [109] and is displayed in Fig. 13 for a rectangular island. The additional potential experienced by the carriers of a given spin confined in the quantum well will, of course, have a similar shape (with the sign reversal for two opposite spin species of the carriers). It suggests that there might be an effective wire formed by spin "up" electrons at the right hand side of the island in the case of the example shown in Fig. 13. Appearance of objects having different nature from that of "ordinary" quantum well excitons were in fact found in microluminescence in the case of iron [110] and cathodoluminescence 


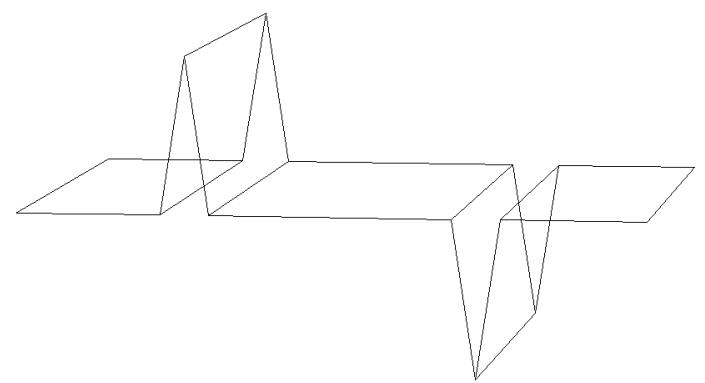

Fig. 13. The profile of the fringe field $z$-component at the distance of $300 \AA$ below the $6 \times 2 \mu \mathrm{m}^{2}$ micromagnet (thickness $200 \mathrm{~nm}$ ) calculated assuming single domain, in-plane magnetization.

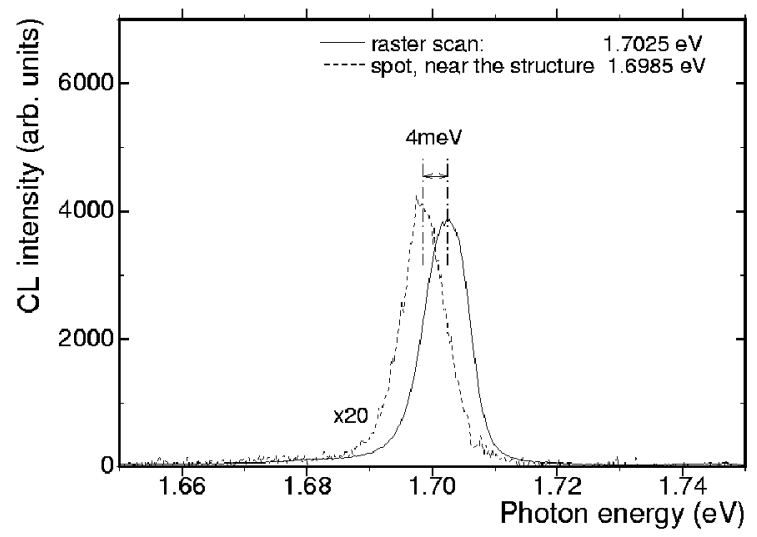

Fig. 14. Cathodoluminescence (CL) spectra in a magnetized sample containing $\mathrm{Cd}_{0.94} \mathrm{Mn}_{0.06}$ Te quantum well buried $300 \AA$ below the surface on which Fe micromagnets were deposited. The averaged spectrum collected from area of approximately $20 \times 20 \mu \mathrm{m}^{2}$ after the raster scan excitation is shown by the solid curve. The broken curve shows the result of point excitation (spot $\approx 1 \mu \mathrm{m}$ in diameter) at the edge of a $6 \times 6 \mu \mathrm{m}^{2}$ micromagnet. $T=10 \mathrm{~K}$, e-beam: $10 \mathrm{kV}, 1.5 \mathrm{nA}$, magnification: $\times 10,000$ [111].

[111]. The latter spectrum observed in the case of a $6 \mu \mathrm{m} \times 6 \mu \mathrm{m}$ iron island atom of $\mathrm{Cd}_{1-x} \mathrm{Mn}_{x} \mathrm{Te}(x=0.06)$ quantum well buried $300 \AA$ in the $\mathrm{CdMgTe}$ cap layer is shown in Fig. 14. In the case of soft magnetic materials a use of a magnetic field is helpful during the experiment. This represents a technical difficulty since either a special immersion lens inserted directly into the liquid helium of a superconducting magnet $[110,112]$ has to be used or specially designed steady magnets can be placed inside a more standard cryostat (see Fig. 15). The disadvantage of the first method is that it is very difficult to scan the sample once it is inserted in the magnet, the disadvantage of the second solution are the limitations of the 


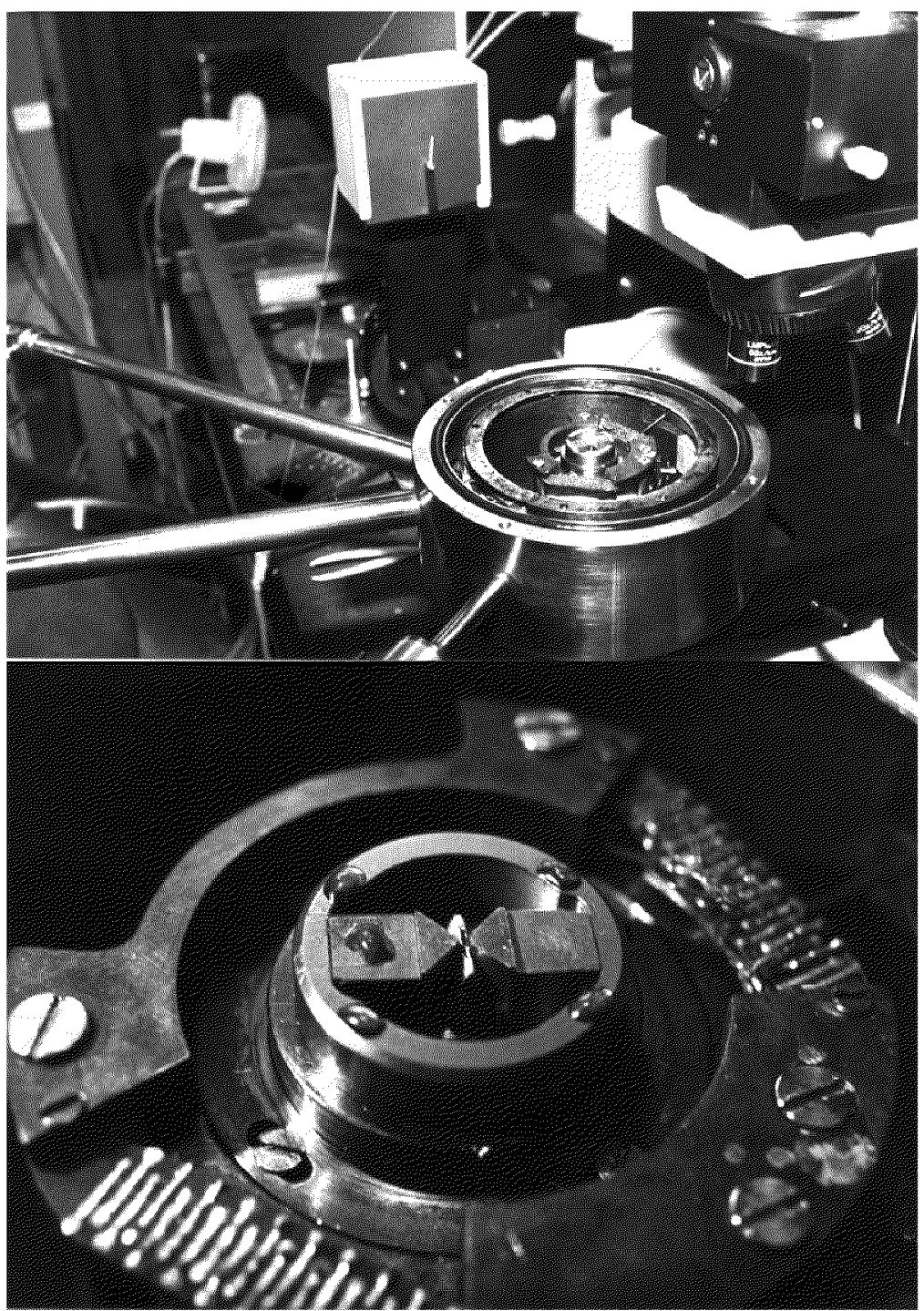

Fig. 15. (a) Home made continuous flow cryostat for microluminescence measurements (with the top plate removed) with a steady NdBFe magnet inserted. The latter, shown in detail in (b), provides the field of $1.3 \mathrm{~T}$ in the slit region between the two shoes.

strength of the field even if modern NdFeB magnets are used (the set up shown in Figs. 15a and $\mathrm{b}$ produces the field of about $1.3 \mathrm{~T}$ ) and the fact that the produced field cannot be varied during the measurements.

Further work is needed, obviously, to optimize the material issues and prove convincingly that the observed additional optical features are indeed related to objects of dimensionality lower than two. 


\section{Acknowledgments}

I am grateful to a numerous group of colleagues whom I collaborated with over a period of over fifteen years on the subject of low-dimensional structures made of diluted magnetic semiconductors. In particular I have to mention M. Aleszkiewicz, J.R. Anderson, W.M. Becker, R. Bylsma, G. Cywiński, M. Czeczott, T. Dietl, P. Dłużewski, M. Dobrowolska-Furdyna, W. Dobrowolski, K. Fronc, J.K. Furdyna, J. Gaj, R.R. Gałązka, M. Ghali, M. Godlewski, G. Grabecki, E. Janik, G. Karczewski, S. Kret, A. Kudelski, M. Kutrowski, F. Kyrychenko, S.H. Lee, G. Lin, H. Luo, S. Maćkowski, I. Merkulov, A. Nakamura, W. Ossau, P. Redliński, N. Samarth, P. Sobkowicz, W. Szuszkiewicz, T. Wojtowicz, J. Wróbel, S. Takeyama, A. Twardowski, D. Yakovlev, I. Yamakawa, without whom the present review would never happen. I am painfully aware that the above list is not exhaustive and I profited from interactions with even greater group of physicists both in Poland and abroad. I am expressing my sincere gratitude to all of them for sharing their wisdom and experience. The $p$-type doping of $\mathrm{ZnTe}, \mathrm{CdTe}$, and related materials was possible owing to the grant from the Foundation for Polish Science for the purchase of nitrogen plasma source for our MBE system (program Techno contract $33 / 2000$ ). The paper was written within the framework of activities of the Centre of Excellence CELDIS funded by EC under contract ICA-CT-2000-70018.

\section{References}

[1] Diluted Magnetic Semiconductors, in series Semiconductors and Semimetals, Eds. J. Kossut, J.K. Furdyna, Vol. 25, Academic Press, San Diego 1987.

[2] J. Kossut, W. Dobrowolski, in: Handbook of Magnetic Materials, Vol. 7, Ed. K.H.J. Buschow, North-Holland, Amsterdam 1993, p. 231.

[3] T. Dietl, in: Handbook of Semiconductors, Vol. 3b, Ed. T.S. Moss, North-Holland, Amsterdam 1994, p. 1251.

[4] J. Kossut, W. Dobrowolski, in: Narrow-Gap II-VI Compounds for Optoelectronic and Electromagnetic Applications, Ed. P. Capper, Chapman and Hall, London 1997, p. 401.

[5] R.R. Gałązka, J. Kossut, T. Story, in: Landoldt Boernstein Numerical Data, Eds. O. Madelung, U. Roessler, M. Schulz, Vol. 41b, Springer Verlag, Berlin 1999, p. 650 .

[6] C. Morrissy, Ph.D. thesis, Oxford University, 1973.

[7] J. Gaj, J. Ginter, R.R. Gałązka, Phys. Status Solidi B 89, 655 (1978).

[8] J. Gaj, R. Planel, G. Fishman, Solid State Commun. 29, 435 (1979).

[9] A.V. Komarov, S.M. Ryabchenko, I.I. Zheru, R.D. Ivanchuk, O.V. Terletskii, $Z h$. Eksp. Teor. Fiz. 74, 608 (1977).

[10] Narrow Gap Semiconductors Physics and Applications, Lecture Notes in Physics, Ed. W. Zawadzki, Vol. 133, Springer, Berlin 1980. 
[11] J.K. Furdyna, J. Kossut, A.K. Ramdas, in: Proc. NATO Advanced Research Workshop, Eds. C.M. Sotomayor Torres, J.C. Portal, J.C. Maan, R.A. Stradling, Plenum Press, New York 1987, p. 135.

[12] J. Kossut, J.K. Furdyna, in: Diluted Magnetic (Semimagnetic) Semiconductors, Eds. R.L. Aggarwal, J.K. Furdyna, S. von Molnar, Material Research Society Symposia Proceeding, Vol. 83, MRS, Pittsburg 1987, p. 97.

[13] J. Kossut, J.K. Furdyna, Acta Phys. Pol. A 73, 851 (1988).

[14] S. Datta, J.K. Furdyna, R.L. Gunshor, Superlattices Microstruct. 1, 327 (1985).

[15] R.N. Bicknell, R.W. Yanka, N.C. Giles-Taylor, D.K. Blanks, E.L. Buckland, J.F. Schetzina, Appl. Phys. Lett. 45, 92 (1984).

[16] L.A. Kolodziejski, T. Sakamoto, R.L. Gunshor, S. Datta, Appl. Phys. Lett. 44, 799 (1984).

[17] P. Kelkar, A.V. Nurmikko, C.-C. Chu, J. Han, W.-L. Chen, R.L. Gunshor, J. Cryst. Growth 184-185, 745 (1998).

[18] H. Luo, J.K. Furdyna, Semicond. Sci. Technol. 10, 1041 (1995).

[19] A.V. Nurmikko, Acta Phys. Pol. A 95, 137 (1999).

[20] H. Ohno, in: The Physics of Semiconductors, Ed. D. Gershoni, World Scientific, Singapore 1999, p. 139.

[21] T. Story, Acta Phys. Pol. A 94, 189 (1998).

[22] T. Story, Acta Phys. Pol. A 98, 171 (2000).

[23] T. Story, Acta Phys. Pol. A 92, 663 (1997).

[24] M.A. Herman, H. Sitter, Molecular Beam Epitaxy. Fundamentals and Current Status, 2nd ed., Springer, Berlin 1996.

[25] G. Grabecki, T. Dietl, J. Kossut, W. Zawadzki, Surf. Sci. 142, 588 (1984).

[26] T. Ando, A.B. Fowler, F. Stern, Rev. Mod. Phys. 54, 437 (1982).

[27] G. Grabecki, T. Dietl, P. Sobkowicz, J. Kossut, W. Zawadzki, Appl. Phys. Lett. 45, 1214 (1984).

[28] G. Karczewski, A.K. Zakrzewski, L. Dobaczewski, W. Dobrowolski, E. Grodzicka, J. Jaroszynski, T. Wojtowicz, J. Kossut, Thin Solid Films 267, 79 (1995).

[29] A.K. Zakrzewski, L. Dobaczewski, G. Karczewski, T. Wojtowicz, J. Kossut, Acta Phys. Pol. A 88, 961 (1995).

[30] E. Janik, E. Dynowska, J. Bąk-Misiuk, T. Wojtowicz, G. Karczewski, J. Kossut, A. Stachow-Wojcik, A. Twardowski, W. Mac, K. Ando, J. Cryst. Growth 184-185, 976 (1998).

[31] T. Wojtowicz, G. Karczewski, J. Kossut, Thin Solid Films 306, 271 (1997).

[32] T. Wojtowicz, M. Kutrowski, G. Karczewski, G. Cywiński, M. Surma, J. Kossut, D.R. Yakovlev, W. Ossau, G. Landwehr, V. Kochereshko, Acta Phys. Pol. A 92, 1063 (1997).

[33] P. Michler, Ed., Phys. Status Solidi B, in press.

[34] E.L. Nagaev, J. Magn. Magn. Mater. 110, 39 (1992).

[35] T. Kasuya, A. Yanase, T. Takeda, Solid State Commun. 8, 1543 (1970).

[36] T. Dietl, J. Spałek, Phys. Rev. B 28, 1548 (1983). 
[37] T. Dietl, J. Spałek, Phys. Rev. Lett. 48, 355 (1982).

[38] M. Nawrocki, R. Planel, G. Fishman, R.R. Gałązka, Phys. Rev. Lett. 46, 735 (1981).

[39] D.U. Bartholomew, J.K. Furdyna, A.K. Ramdas, Phys. Rev. B 34, 6943 (1986).

[40] E. Oh, D.U. Bartholomew, A.K. Ramdas, J.K. Furdyna, U. Debska, Phys. Rev. B 38, 13183 (1988).

[41] C. Benoit à la Guillaume, Phys. Status Solidi B 175, 369 (1993).

[42] C. Benoit à la Guillaume, Mater. Sci. Forum 182-184, 549 (1995).

[43] R.B. Bylsma, W.M. Becker, J. Kossut, U. Debska, D. Yoder-Short, Phys. Rev. B 33, 8207 (1986).

[44] D.R. Yakovlev, W. Ossau, G. Landwehr, R.N. Bicknell-Tassius, Solid State Commun. 76, 325 (1990).

[45] A.K. Bhattacharjee, C. Benoit à la Guillaume, Phys. Rev. B 55, 10613 (1997).

[46] G. Mackh, M. Hilpert, D.R. Yakovlev, W. Ossau, H. Heinke, T. Litz, F. Fisher, A. Waag, G. Landwehr, R. Hellmann, E.O. Goedel, Phys. Rev. B 50, 14069 (1994).

[47] N. Takahashi, K. Takabayashi, E. Shirado, I. Souma, J.X. Shen, Y. Oka, J. Cryst. Growth 214/215, 183 (2000).

[48] J.A. Gaj, R.R. Gałązka, M. Nawrocki, Solid State Commun. 25, 193 (1978).

[49] K. Ando, T. Okoshi, N. Koshizuka, Appl. Phys. Lett. 53, 4 (1988).

[50] K. Nakamura, H. Nakano, J. Phys. Soc. Japan Lett. 59, 1154 (1990).

[51] W. Maślana, W. Mac, J.A. Gaj, P. Kossacki, A. Golnik, J. Cibert, S. Tatarenko, T. Wojtowicz, G. Karczewski, J. Kossut, Phys. Rev. B 63, 165318 (2001).

[52] M. von Ortenberg, Phys. Rev. Lett. 49, 1041 (1982).

[53] X. Liu, A. Petrou, J. Warnock, B.T. Jonker, G.A. Prinz, J.J. Krebs, Phys. Rev. B 63, $2280(1989)$.

[54] N. Dai, H. Luo, F.C. Zhang, N. Samarth, M. Dobrowolska, J.K. Furdyna, Phys. Rev. Lett. 67, 3824 (1991).

[55] W.C. Chou, A. Petrou, J. Warnock, B.T. Jonker, Phys. Rev. Lett. 67, 3820 (1991).

[56] J.A. Brum, G. Bastaed, M. Voos, Solid State Commun. 59, 2280 (1986).

[57] E. Deleporte, J.M. Berroir, G. Bastard, C. Delalande, J.M. Hong, L.L. Chang, Phys. Rev. B 42, 5891 (1990).

[58] H.H. Cheng, R.J. Nicholas, D.E. Ashenford, B. Lunn, Phys. Rev. B 56, 10453 (1997).

[59] G. Yang, J.K. Furdyna, H. Luo, Phys. Rev. B 67, 4226 (2000).

[60] N. Dai, L.R. Ram-Mohan, H. Luo, G.L. Yang, F.C. Zhang, M. Dobrowolska, J.K. Furdyna, Phys. Rev. B 50, 18153 (1994).

[61] G. Prechtl, W. Heiss, A. Bonanni, W. Jantsch, S. Mackowski, E. Janik, G. Karczewski, Phys. Rev. B 61, 15617 (2000).

[62] J.A. Gaj, W. Grieshaber, C. Bodin-Deshayes, J. Cibert, G. Feuillet, Y. Merle d'Aubigné, A. Wasiela, Phys. Rev. B 50, 5512 (1994).

[63] W. Grieshaber, A. Haury, J. Cibert, Y. Merle d'Aubigne, A. Wasiela, J.A. Gaj, Phys. Rev. B 53, 4891 (1996). 
[64] I. Lawrence, S. Haacke, H. Mariette, W.W. Ruehle, H. Ulmer-Tuffigo, J. Cibert, G. Feuillet, Phys. Rev. Lett. 73, 2131 (1994).

[65] C.R. Becker, X.C. Zhang, K. Ortner, J. Schmidt, A. Pfeuffer-Jeschke, V. Latussek, Y.S. Gui, V. Daumer, J. Liu, H. Buhman, G. Landwehr, L.W. Molenkamp, Thin Solid Films, in press.

[66] Y.D. Kim, M.V. Klein, S.F. Ren, Y.C. Chang, H. Luo, N. Samarth, J.K. Furdyna, Phys. Rev. B 49, 7262 (1994).

[67] S.M. Durbin, J. Han Sungki, O.M. Kobayashi, D.R. Menke, R.L. Gunshor, Q. Fu, N. Pelekanos, A.V. Nurmikko, D. Li, J. Gonsalves, N. Otsuka, Appl. Phys. Lett. 55, 2087 (1989).

[68] E. Janik, E. Dynowska, J. Bąk-Misiuk, M. Leszczyński, W. Szuszkiewicz, T. Wojtowicz, G. Karczewski, A.K. Zakrzewski, J. Kossut, Thin Solid Films 267, 74 (1995).

[69] K. Ando, K. Takahashi, T. Okuda, Phys. Rev. B 46, 12289 (1992).

[70] P.W. Anderson, Phys. Rev. 79, 705 (1950).

[71] J. Pietruczanis, W. Mac, A. Twardowski, G. Karczewski, A. Zakrzewski, E. Janik, T. Wojtowicz, J. Kossut, Mater. Sci. Forum 182-184, 687 (1995).

[72] A. Stachow-Wojcik, W. Mac, A. Twardowski, G. Karczewski, E. Janik, T. Wojtowicz, J. Kossut, E. Dynowska, Phys. Status Solidi A 177, 555 (2000).

[73] M. Jouanne, W. Szuszkiewicz, J.F. Morhange, M.A. Kanehisa, J.M. Hartmann, H. Mariette, E. Dynowska, G. Karczewski, T. Wojtowicz, J. Kossut, J. Barnaś, J. Cryst. Growth 184-185, 947 (1998).

[74] W. Szuszkiewicz, M. Jouanne, E. Dynowska, E. Janik, G. Karczewski, T. Wojtowicz, J. Kossut, Acta Phys. Pol. A 88, 941 (1995).

[75] E. Dynowska, E. Janik, J. Bak-Misiuk, J. Domagala, T. Wojtowicz, J. Kossut, J. Alloys Comp. 286, 276 (1999).

[76] A.C. Gossard, M. Sundaram, P.F. Hopkins, in: Epitaxial Microstructures, Ed. A.C. Gossard, Vol. 40 of Semiconductors and Semimetals, Academic Press, Boston 1994, p. 153.

[77] T. Wojtowicz, M. Kutrowski, G. Karczewski, J. Kossut, Acta Phys. Pol. A 94, 199 (1998)

[78] M. Kutrowski, T. Wojtowicz, S. Kret, G. Karczewski, J. Kossut, R. Fiederling, B. Koenig, D.R. Yakovlev, W. Ossau, A. Waag, V.P. Kochereshko, F.J. Teran, M. Potemski, in: Optical Properties of Semiconductor Nanostructures, Eds. M.L. Sadowski, M. Potemski, M. Grynberg, Kluwer Academic Publ., Dordrecht 2000, p. 237.

[79] P.A. Wolff, L.R. Ram-Mohan, in: Diluted Magnetic (Semimagnetic) Semiconductors, Eds. R.L. Aggarwal, J.K. Furdyna, S. von Molnar, Material Research Society Symposia Proceedings, Vol. 89, MRS, Pittsburgh 1987, p. 1.

[80] M. Kutrowski, G. Karczewski, G. Cywiński, M. Surma, K. Grasza, E. Łusakowska, J. Kossut, T. Wojtowicz, R. Fiederling, D.R. Yakovlev, G. Mackh, U. Zehnder, W. Ossau, Thin Solid Films 306, 283 (1997).

[81] D. Wasik, J. Przybytek, M. Baj, G. Karczewski, T. Wojtowicz, J. Kossut, Mater. Sci. Forum 182-184, 247 (1995). 
[82] D. Wasik, K. Kudyk, M. Baj, J. Jaroszyński, T. Wojtowicz, A. Barcz, Phys. Rev. $B$ 89, 12917 (1999).

[83] H. Luo, J.K. Furdyna, B. McCombe, private communication.

[84] G. Karczewski, J. Jaroszynski, A. Barcz, M. Kutrowski, T. Wojtowicz, J. Kossut, J. Cryst. Growth 184/185, 814 (1998)

[85] H. Kunimatsu, S. Takeyama, K. Uchida, N. Miura, G. Karczewski, T. Wojtowicz, J. Kossut, Physica B 249-251, 951 (1998).

[86] Y. Imanaka, T. Takamasu, G. Kido, G. Karczewski, T. Wojtowicz, J. Kossut, Physica E 10, 336 (2001).

[87] S. Crooker, N. Samarth, D.D. Awschalom, Phys. Rev. B 61, 1736 (2000).

[88] C.S. Kim, M. Kim, J.K. Furdyna, M. Dobrowolska, S. Lee, L.M. Smith, H.E. Jackson, E.M. James, Y. Xin, N.D. Browning, Phys. Rev. Lett. 85, 1124 (2001).

[89] T. Wojtowicz, M. Kutrowski, G. Karczewski, J. Kossut, F.J. Teran, M. Potemski, Phys. Rev. B 59, 10437 (1999).

[90] I.A. Merkulov, D.R. Yakovlev, A. Keller, W. Ossau, J. Geurts, A. Waag, G. Landwehr, G. Karczewski, T. Wojtowicz, J. Kossut, Phys. Rev. Lett. 83, 1431 (1999).

[91] A.K. Bhattacharjee, Phys. Rev. B 58, 15660 (1998).

[92] T. Wojtowicz, M. Kutrowski, G. Karczewski, J. Kossut, Appl. Phys. Lett. 73, 1379 (1998)

[93] Y. Ohno, D. Young, B. Beschoten, F. Matsukura, H. Ohno, D.D. Awschalom, Nature 402, 790 (1999).

[94] R. Fiederling, M. Kelm, G. Reuscher, W. Ossau, G. Schmidt, A. Waag, L. Molenkamp, Nature 402, 787 (1999).

[95] M. Oestreich, J. Hubner, D. Hagele, P.J. Klar, W. Hembrodt, W.W. Ruhle, Appl. Phys. Lett. 74, 1251 (1999).

[96] M. Ghali, J. Kossut, E. Janik, K. Reginski, L. Klopotowski, Solid State Commun. 119, 371 (2001).

[97] B.T. Jonker, Y.D. Park, B.R. Bennet, H.D. Cheong, G. Kioseoglou, A. Petrou, Phys. Rev. B 62, 8180 (2000).

[98] B.T. Jonker, A. Hanbicki, Y.D. Park, G. Itskos, M. Furis, G. Kioseoglou, A. Petrou, X. Wei, Appl. Phys. Lett. 79, 3098 (2001).

[99] J. Carlos Egues, Phys. Rev. Lett. 80, 4578 (1998).

[100] M. Brun, S. Huant, J.-C. Woehl, J.-F. Motte, L. Marsal, H. Mariette, J. Microscopy 202, 202 (2001).

[101] L. Besombes, K. Kheng, L. Marsal, H. Mariette, Phys. Rev. B 63, 155307 (2001).

[102] S. Maćkowski, G. Karczewski, T. Wojtowicz, J. Kossut, S. Kret, A. Szczepańska, P. Dłużewski, G. Prechtl, W. Heiss, Appl. Phys. Lett. 78, 3884 (2001).

[103] S. Maćkowski, J. Wrobel, K. Fronc, J. Kossut, F. Pulizzi, P.C.M. Christiannen, J.C. Maan, G. Karczewski, Phys. Status Solidi B, in press.

[104] G. Bacher, A.A. Maksimov, A. McDonald, H. Schoemig, M.K. Welsch, V.D. Kulakovskii, A. Forchel, C.R. Becker, L.W. Molenkamp, G. Landwehr, Phys. Status Solidi $B$ 224, 573 (2001). 
[105] A.A. Maksimov, G. Bacher, A. McDonald, V.D. Kulakovskii, A. Forchel, C.R. Becker, G. Landwehr, L.W. Molenkamp, Phys. Rev. B 62, 7767 (2000).

[106] S. Maćkowski, S. Lee, J.K. Furdyna, M. Dobrowolska, G. Prechtl, W. Heiss, J. Kossut, G. Karczewski, Phys. Status Solidi B, in press.

[107] T. Dietl, J. Magn. Magn. Mater. 38, 34 (1983).

[108] G.A. Prinz, Science 250, 1097 (1990).

[109] J. Reijners, F.M. Peeters, A. Matulis, Phys. Rev. B 59, 2817 (1999).

[110] A. Kudelski, K. Fronc, J. Wróbel, S. Maćkowski, G. Cywiński, M. Aleszkiewicz, F. Kyrychenko, T. Wojtowicz, J. Kossut, J. Gaj, Solid State Commun. 120, 35 (2001)

[111] J. Kossut, I. Yamakawa, A. Nakamura, G. Cywiński, K. Fronc, M. Czeczott, J. Wróbel, F. Kyrychenko, T. Wojtowicz, S. Takeyama, Appl. Phys. Lett. 79, 1789 (2001).

[112] J. Jasny, J. Sepiol, Chem. Phys. Lett. 273, 439 (1997).

[113] M. Kutrowski, Ph.D. thesis, 2001, unpublished. 